\title{
Evaluación económica para Colombia de las principales intervenciones farmacológicas como monoterapia para el tratamiento de la hipertensión arterial leve a moderada recién diagnosticada
}

\author{
Economic evaluation for Colombia of the main \\ pharmacological interventions as monotherapy \\ for the treatment of newly diagnosed mild to \\ moderate hypertension
}

\author{
Rodolfo J. Dennis, María X. Rojas, Martín Rondón, Ludy A. Parada, \\ Juan Carlos Villar • Bogotá, D.C. (Colombia)
}

\section{Resumen}

Objetivo: en el año 2012 se desarrolló para Colombia la guía de práctica clínica para manejo de la hipertensión arterial (HTA). Esta evaluación económica (EE) fue desarrollada para identificar la costo-efectividad de los diferentes tratamientos farmacológicos usados como monoterapia, y soportar las recomendaciones en la guía.

Diseño: se simuló una cohorte de pacientes adultos, con diagnóstico de hipertensión arterial. La EE se basó en un modelo desarrollado para el National Institute for Clinical Excellence (NICE) del Reino Unido. Este modelo fue adaptado para Colombia en consumo de recursos y costos. La efectividad de las intervenciones y probabilidades de transición fueron actualizadas para 2012.

Resultados: los resultados del análisis del caso de referencia (hombre y mujer de 65 años, $2 \%$ de riesgo anual de enfermedad CV, $1 \%$ de falla cardiaca, $1.1 \%$ de diabetes, 20 años de seguimiento), mostraron que los años de vida ajustados por calidad (QALY) obtenidos con la terapia de diuréticos, inhibidores de enzima convertidora/bloqueadores del receptor de angiotensina (ACEi/ARB) y calcioantagonistas fue 9.24, 9.24 y 9.26, respectivamente. Los costos favorecen a los diuréticos (COP \$6 498 624), en comparación con ACEi/ARB o calcioantagonistas (COP \$13 178 919, y COP \$27 774 098), respectivamente. La terapia con betabloqueadores y la no intervención fueron dominadas.

Conclusiones: la opción de tratamiento con diuréticos tipo tiazidas como mono-terapia inicial es la más costo-efectiva. La principal diferencia entre las alternativas está dada especialmente por las diferencias en el costo de los medicamentos, ya que la efectividad es similar entre los tratamientos con diuréticos y ACEi/ARB, y sólo discretamente mejor con calcio-antagonistas. (Acta Med Colomb 2015; 40: 279-287).

Palabras clave: hipertensión, costos, eficacia, evaluación económica, drogas.

\section{Abstract}

Objective: in 2012 the guide of clinical practice for management of arterial hypertension (AHT) was developed for Colombia. This economic evaluation (EE) was developed to identify the costeffectiveness of different pharmacological treatments used as monotherapy, and to support the recommendations in the guidelines.

Design: a cohort of adult patients with a diagnosis of hypertension was simulated. The EE was based on a model developed for the National Institute for Clinical Excellence (NICE) in the UK.
Dr. Rodolfo J. Dennis, MSc FACP: Departamento de Investigaciones, Fundación Cardioinfantil - Instituto de Cardiología. Departamento de Epidemiología Clínica y Bioestadística, Facultad de Medicina, Pontificia Universidad Javeriana. Unidad de Unidad de Síntesis y Transferencia de Conocimiento, Programa CARDIECOL; Dra. María X. Rojas, RN, MSc PhD.: Departamento de Epidemiología Clínica y Bioestadística, Facultad de Medicina, Pontificia Universidad Javeriana; Dr. Martín Rondón, MSc.: Departamento de Epidemiología Clínica y Bioestadística, Facultad de Medicina, Pontificia Universidad Javeriana; Dra. Ludy A. Parada, Mg.: Departamento de Epidemiología Clínica y Bioestadística, Facultad de Medicina, Pontificia Universidad Javeriana; Dr. Juan Carlos Villar, MSc PhD.: Departamento de Investigaciones, Fundación CardioinfantilInstituto de Cardiología. Departamento de Medicina Interna, Facultad de Ciencias de la Salud, Universidad Autónoma de Bucaramanga. Unidad de Unidad de Síntesis y Transferencia de Conocimiento, Programa CARDIECOL. Bogotá, D.C. (Colombia).

Correspondencia. Dr. Rodolfo J. Dennis. Bogotá, D.C. (Colombia).

E-mail: rdennis@cardioinfantil.org

Recibido: 28/I/2015 Aceptado: 20/VIII/2015 
This model was adapted for Colombia in resource consumption and costs. The effectiveness of interventions and transition probabilities were updated for 2012.

Results: the results of the analysis of the reference case (man and woman of $65.2 \%$ annual risk of cardiovascular disease, $1 \%$ of heart failure, $1.1 \%$ of diabetes, 20 years of follow-up) showed that quality-adjusted life year (QALY) obtained with diuretic therapy, converting enzyme inhibitors / angiotensin receptor blockers (ACEi / ARB) and calcium antagonists was 9.24, 9.24 and 9.26, respectively. Costs favor diuretics (COP \$6 498 624) compared with ACEi / ARB or calcium antagonists (COP \$ 13178919 and \$ 27774098 COP), respectively. Therapy with beta-blockers and non-intervention were dominated.

Conclusions: the choice of treatment with thiazide-type diuretics as initial monotherapy is the most cost-effective. The main difference between alternatives is given especially by differences in drug costs, since the effectiveness is similar between treatments with diuretics and ACEi / ARB, and only slightly better with calcium antagonists. (Acta Med Colomb 2015; 40: 279-287).

Key Words: hypertension, costs, effectiveness, economic evaluation, drugs.

\section{Introducción}

La hipertensión arterial (HTA) es el factor de riesgo que explica la mayor cantidad de muertes asociadas con desenlaces cardiovasculares en el mundo (1). La carga global de enfermedad asociada con la HTA es responsable de siete millones de muertes prematuras, y de aproximadamente 92 millones de años de vida perdidos por discapacidad, $6 \%$ de la carga total de enfermedad en el mundo (2). Latinoamérica tiene de las prevalencias de HTA más altas del mundo, con poco conocimiento en promedio de la enfermedad, y superando $40 \%$ de prevalencia en hombre adultos (3-4). Llama la atención que el principal problema actual en el tratamiento de esta condición tiene que ver con la adherencia al manejo y no con ausencia de medicamentos altamente efectivos y costo-efectivos (5-7).

En el año 2010 el Ministerio de Salud y Protección Social (MSPS) comisionó a través de la convocatoria 500 de 2010 de Colciencias, una serie de guías de práctica clínica, incluyó una sobre la prevención, tratamiento y seguimiento de la hipertensión arterial primaria. En los siguientes dos años el grupo desarrollador produjo esta guía (8) que incluyó 34 recomendaciones basadas en 24 preguntas estructuradas, proceso que siguió los lineamientos de la guía metodológica colombiana para estos documentos (9). Una de estas preguntas se refería a la mejor opción para iniciar tratamiento farmacológico con monoterapia con las principales intervenciones farmacológicas disponibles para el tratamiento de la HTA leve a moderada recién diagnosticada. Dado el impacto en el gasto de salud que tiene una enfermedad como la hipertensión arterial se consideró altamente relevante, en consenso con el Ministerio de Salud de Colombia, realizar un análisis económico de costo-efectividad que permitiera identificar, entre las opciones de tratamiento actualmente disponibles en el país para el manejo de la HTA, cuál o cuáles son las alternativas de manejo farmacológico más adecuadas para ser recomendadas como manejo inicial, por estar asociadas con mejores desenlaces en salud y con menores costos. Sin duda adelantar una EE en este aspecto era relevante, dado que las alternativas farmacológicas enfrentadas afectan desenlaces críticos (eventos cardiovasculares mayores), hay suficiente evidencia de elevada calidad respecto a la eficacia y efectividad de las cinco principales alternativas farmacológicas como para informar el proceso de análisis económico, no hay clara evidencia a priori de la dominancia de una alternativa, y hay elevada variabilidad en la práctica actual en la escogencia de las alternativas farmacológicas enfrentadas (10). Por ende, la identificación de la(s) alternativas más costo-efectivas podría ayudar a racionalizar sustancialmente la utilización de recursos y contener costos.

Este artículo presenta el abordaje realizado para el estudio de costo-efectividad y sus resultados.

\section{Material y métodos}

Se diseñó un análisis de costo-efectividad que permitiera comparar las alternativas de tratamiento farmacológico usadas como monoterapia en el tratamiento de pacientes adultos con hipertensión arterial primaria no controlada con intervenciones no farmacológicas. Se tomó como base el modelo realizado por el National Institute of Clinical Excellence (NICE) del Reino Unido, para responder la misma pregunta como parte de la guía \#34, titulada "Clinical management of primary hypertension in adults" (11).

\section{Población objeto de estudio}

Se modeló una cohorte hipotética de pacientes adultos (hombres, mujeres) de 45 años de edad o más, con diagnóstico reciente de hipertensión arterial, que no respondieron favorablemente (control de la HTA) a cambios en el estilo de vida (dieta y ejercicio) y que adicionalmente no tenían diagnóstico previo de enfermedad cardiovascular, falla cardiaca y diabetes.

\section{Alternativas comparadas}

Con base en las guías contemporáneas de práctica clínica (11), y la nueva guía colombiana (8), se determinó comparar 
en el modelo los tratamientos basados en: diuréticos (D); bloqueadores de los canales del calcio/calcioantagonistas (C); betabloqueadores (B); antagonistas del receptor de angiotensina (ARA) e inhibidores de la enzima convertidora de angiotensina (IECA) (A). Adicionalmente se incluyó la "no intervención como una opción terapéutica, únicamente a título de comparación. Se incluyeron los ARA y los IECA en un solo grupo comparador dada su aparente intercambiabilidad al obtener resultados clínicos similares. Teniendo en cuenta la posible intolerancia a los IECA reportada en la literatura, se consideró que $80 \%$ de los pacientes del grupo recibirían tratamiento con un IECA y $20 \%$ con un ARA.

\section{Desenlaces}

La efectividad del tratamiento se midió por el impacto en la frecuencia de presentación de angina inestable (AI), infarto agudo de miocardio (IAM), falla cardiaca, accidente cerebrovascular y muerte relacionada con enfermedad cardiovascular (ECV), como desenlaces primarios. Como desenlaces secundarios se incluyeron los efectos secundarios más comúnmente relacionados con el tratamiento anti-hipertensivo, como la aparición de falla cardiaca y la diabetes tipo II. Como medida de utilidad al final de la vida se tomó "años de vida ajustados por calidad" (QALY de sus siglas en inglés quality adjusted life years). El principal desenlace económico fue el "costo incremental por QALY" con cada tratamiento al final de la vida, comparado con la opción de menor costo [razón de costo efectividad incremental (ICER) de sus siglas en inglés].

\section{Perspectiva}

El análisis se consideró desde la perspectiva del Sistema General de Seguridad Social en Salud (pagador). Por tanto, se incluyeron únicamente los costos médicos directos.

\section{Horizonte de tiempo}

Como la HTA tiene impacto en la morbilidad a largo plazo y consecuencias en la mortalidad, para el resultado primario del modelo se asumió un horizonte de tiempo hasta el final de la vida. La tasa de descuento usada para costos y desenlaces corresponde a una tasa constante de $3 \%$ anual, de acuerdo con la recomendación del Panel de Washington (12).

\section{Información sobre efectividad y seguridad}

Las probabilidades de eventos cardiovasculares para la población de 45 y 55 años fueron estimados a partir de los riesgos de eventos reportados en la literatura (ver "estructura y consideraciones del modelo"). Para la estimación de la mortalidad relacionada con ECV por edad y sexo se tomaron datos reportados por el Departamento Administrativo Nacional de Estadística (DANE) de Colombia para el año 2008 (13).

\section{Estimación de utilidades y QALYs}

Dado que para Colombia no se cuenta con información válida acerca de las preferencias de la población sobre los diferentes estados de salud, el grupo evaluador, después de considerar las diferentes opciones disponibles, decidió utilizar para el cálculo de QALYS en este análisis, la asignación de las utilidades asociadas a los diferentes estados de salud de la enfermedad cardiovascular y la HTA que fueron usadas para el estudio realizado por Ward en 2005 (ScHARR-TAG 2005). Estas utilidades han sido usadas en la mayor parte de los estudios económicos del área cardiovascular que hacen parte del registro "The Cost-Effectiveness Analysis Registry" del "Center for the Evaluation of Value and Risk in Health" del "Institute for Clinical Research and Health Policy Studies" (Tufts Medical Center) (14). Para la diabetes, la ECV e insuficiencia cardiaca, se supuso una utilidad constante desde el inicio de la enfermedad. Las utilidades fueron ajustadas para reflejar el hecho de que la calidad de vida relacionada con salud en la población general disminuye con la edad.

\section{Estimación y valoración del consumo de recursos}

Para identificar y medir el consumo de recursos asociados a la atención hospitalaria de los desenlaces AI, IAM, ECV etc., se partió de la lista de recursos considerados en el modelo original desarrollado por NICE, la cual fue validada en un consenso informal de expertos del grupo desarrollador de la guía de HTA para Colombia. Para identificar y medir el consumo de recursos en el tratamiento ambulatorio, se buscó llegar a un consenso de expertos aplicando la metodología DELPHI. Una vez identificados los recursos, para su valoración se usaron las tarifas del Instituto de Seguros Sociales (ISS) del año 2001 (15), ajustadas en 30\% adicional al valor definido para el caso base, con el fin de aproximarlas al valor presente para Colombia en 2012, y con unos rangos de 25 y $48 \%$ adicional para los análisis de sensibilidad.

\section{Estimación del costo de los medicamentos}

Para la estimación de costos de los medicamentos, se siguió la metodología de costeo descrita en el artículo 1 del Decreto 4474 de 2010 (16). Se tomaron las bases de datos del Sistema Integral de Información de la Protección Social (SISPRO) y específicamente del Sistema de Información de Precios de Medicamentos (SISMED) del Ministerio de la Protección Social.

\section{Estructura y consideraciones del modelo}

Se consideró un modelo de Markov para evaluar las diferencias en los costos y desenlaces del tratamiento a largo plazo de la HTA leve a moderada, entre los diferentes grupos de medicamentos antihipertensivos de primera línea en cuidado primario, y la opción de no tratamiento, desde la perspectiva del Sistema General de Seguridad Social en Salud. Considerando la historia natural de la enfermedad, en el modelo, todos los pacientes hipertensos empiezan en un estado libre de desenlaces. Los ciclos de tránsito entre los diferentes desenlaces fueron modelados cada seis meses, donde una proporción importante de los pacientes 
hipertensos se mantendrían libre de eventos. Los pacientes pueden experimentar más de un evento no fatal en los periodos siguientes del modelo. Todos los pacientes terminan en el modelo en un estado de muerte, no obstante, dadas las características de un modelo de Markov, al final una cierta proporción de la cohorte continúa con vida, no importa que tan alta sea la tasa de mortalidad. La Figura 1 muestra una representación esquemática del modelo. En él se asumió que la HTA (sola sin otros desenlaces concomitantes) no aumenta el riesgo muerte comparada con la mortalidad de la población general. Los riesgos de mortalidad y otros eventos cardiovasculares en los pacientes que desarrollan diabetes se supuso que era el doble de los observados en pacientes no diabéticos.

La estructura conceptual del modelo fue validada por consulta a expertos clínicos del grupo desarrollador de la guía (GDG) y a expertos locales en evaluación económica, así como a representantes del Ministerio de Salud. El modelo incorpora datos específicos para Colombia en términos de expectativa de vida, mortalidad de causa cardiovascular y no cardiovascular, costos asociados con el precio de los medicamentos, y consumo de recursos asociado con las consecuencias del tratamiento (desenlaces).

\section{Información en el modelo}

Se incluyeron las variables clínicas (eventos cardiovasculares, riesgo basal de falla cardiaca, riesgo basal de diabetes, riesgo de muerte), derivada de estudios clínicos (17-20); las variables de los costos (costos del tratamiento farmacológico, costos del manejo ambulatorio del paciente hipertenso con y sin eventos cardiovasculares previos, costos hospitalarios de la atención de eventos cardiovasculares), y las variables de la entrada de la utilidad asociada a cada estado de salud en el modelo. Las probabilidades de transición anuales para hombres y mujeres de 65 años usadas en el modelo se presentan en las Tablas 1 ( $\mathrm{a}$ y b) y 2 ( $\mathrm{a}$ y b).

Los efectos relativos de tratamiento se basan en las estimaciones (metaanálisis) realizados por NICE a partir de la revisión sistemática de la literatura adelantada para desarrollar el modelo original sobre estudios que compararon las diferentes grupos de medicamentos contra placebo y frente a frente. Estos datos fueron actualizados para el análisis económico actual para Colombia; todos estos estudios fueron incluidos para estimar los efectos del tratamiento de los cuatro grupos de medicamentos frente a la alternativa de "No intervención", basados en comparaciones directas e indirectas (Tabla 3).

\section{Definición del caso de referencia (caso base)}

Se consideraron dos cohortes, una de hombres y otra de mujeres, de 65 años, que al inicio del tratamiento presentaban $2 \%$ de riesgo anual de enfermedad $\mathrm{CV}, 1 \%$ de falla cardiaca, $1.1 \%$ de diabetes mellitus tipo 2 , y asumiendo que $20 \%$ de los sujetos serán intolerantes a IECA y necesitarán un ARA. Los pacientes en la cohorte fueron seguidos por

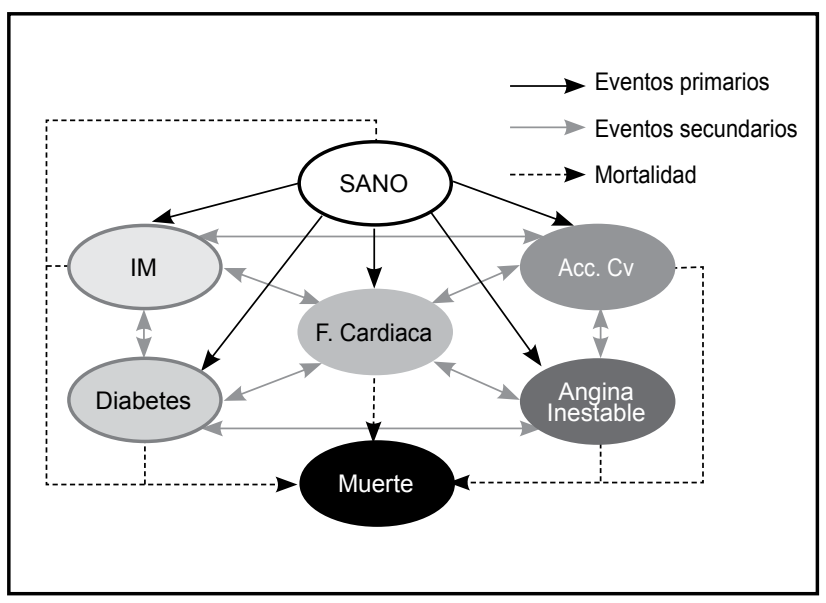

Figura 1. Estructura del modelo de Markov para evaluar la costo-efectividad de los medicamentos para el tratamiento de la hipertensión arterial. IM: infarto miocárdico; F Cardíaca: falla cardiaca; Acc.Cv: accidente cerebrovascular. Fuente: esquema proporcionado por el grupo desarrollador del modelo en NICE, UK.

Tabla 1. Probabilidad de transición para pacientes hombres de 65 años no tratados (riesgo anual de enfermedad cardiovascular [CVD] de $2 \%$ )

\begin{tabular}{|c|c|c|}
\hline Parámetro & $\begin{array}{l}\text { Probabilidad } \\
\text { anual }(\%)\end{array}$ & Fuente \\
\hline Sano a angina inestable & 0.0017 & ScHARR-TAG 2005 (16) \\
\hline Sano a IM & 0.0035 & ScHARR-TAG 2005 \\
\hline Sano a diabetes & 0.0110 & ASCOT 2005 (17) \\
\hline Sano a Acc.Cv & 0.0054 & ScHARR-TAG 2005 \\
\hline Sano a falla cardiaca & 0.0098 & SHEP 2000 (18) \\
\hline Sano a muerte & 0.0180 & Tablas de vida de la población \\
\hline Angina inestable a IM & 0.0300 & ScHARR-TAG 2005 \\
\hline Angina inestable a diabetes & 0.0067 & Se asume igual que de IM a diabetes \\
\hline Angina inestable a Acc.Cv & 0.0095 & Se asume que es igual que IM a $\mathrm{Acc} . \mathrm{Cv}$ \\
\hline $\begin{array}{l}\text { Angina inestable } \\
\text { a falla cardiaca }\end{array}$ & 0.0230 & Se asume que es igual que IM a falla \\
\hline Angina inestable a muerte & 0.0348 & $\begin{array}{l}\text { ScHARR-TAG } 2005 \text { y tablas de vida } \\
\text { de la población }\end{array}$ \\
\hline IM a angina inestable & 0.0078 & HOPE trial 2000 (19) \\
\hline IM a otro IM & 0.0721 & ScHARR-TAG 2005 \\
\hline IM a diabetes & 0.0067 & HOPE trial 2000 \\
\hline IM a Acc.Cv & 0.0095 & ScHARR-TAG 2005 \\
\hline IM a falla cardiaca & 0.0230 & HOPE trial 2000 \\
\hline IM a muerte & 0.0258 & $\begin{array}{l}\text { ScHARR-TAG } 2005 \text { y tablas de vida } \\
\text { de la población }\end{array}$ \\
\hline Diabetes a angina inestable & 0.0033 & Doble riesgo de población sana \\
\hline Diabetes a IM & 0.0069 & Doble riesgo de población sana \\
\hline Diabetes a Acc.Cv & 0.0108 & Doble riesgo de población sana \\
\hline Diabetes a falla cardiaca & 0.0197 & Doble riesgo de población sana \\
\hline Diabetes a muerte & 0.0359 & Doble riesgo de población sana \\
\hline Acc. $\mathrm{Cv}$ a angina inestable & 0.0016 & Se asume que es igual que Acc.Cv a IM \\
\hline Acc.Cv a IM & 0.0016 & ScHARR-TAG 2005 \\
\hline Acc.Cv a diabetes & 0.0067 & Se asume que es igual que de IM a diabetes \\
\hline Acc.CV a Acc.Cv & 0.2875 & ScHARR-TAG 2005 \\
\hline Acc. $\mathrm{Cv}$ a falla cardiaca & 0.0115 & $\begin{array}{l}\text { Se asume que es la mitad de IM } \\
\text { a falla cardiaca }\end{array}$ \\
\hline Acc. $\mathrm{Cv}$ a muerte & 0.3548 & $\begin{array}{l}\text { ScHARR-TAG } 2005 \text { y tablas de vida } \\
\text { de la población }\end{array}$ \\
\hline
\end{tabular}


Tabla 2. Probabilidad de transición para mujeres de 65 años no tratadas (riesgo anual de enfermedad cardiovascular [CVD] $2 \%$ ).

\begin{tabular}{|c|c|c|}
\hline Parámetro & $\begin{array}{c}\text { Probabilidad } \\
\text { anual }\end{array}$ & Fuente \\
\hline Sano a angina inestable & 0.0010 & ScHARR-TAG 2005 (16) \\
\hline Sano a IM & 0.0021 & ScHARR-TAG 2005 \\
\hline Sano a diabetes & 0.0110 & ASCOT 2005 (17) \\
\hline Sano a Acc.Cv & 0.0076 & ScHARR-TAG 2005 \\
\hline Sano a falla cardiaca & 0.0098 & SHEP 2000 (18) \\
\hline Sano a muerte & 0.0141 & $\begin{array}{l}\text { ScHARR-TAG } 2005 \text { y tablas de vida de } \\
\text { la población }\end{array}$ \\
\hline Angina inestable a IM & 0.0300 & ScHARR-TAG 2005 \\
\hline Angina inestable a diabetes & 0.0067 & Se asume que es igual que IM a diabetes \\
\hline Angina inestable a Acc.Cv & 0.0095 & Se asume que es igual que IM a Acc.Cv \\
\hline $\begin{array}{l}\text { Angina inestable a } \\
\text { falla cardiaca }\end{array}$ & 0.0230 & $\begin{array}{l}\text { Se asume que es igual que IM a falla } \\
\text { cardiaca }\end{array}$ \\
\hline Angina inestable a muerte & 0.0307 & $\begin{array}{l}\text { ScHARR-TAG } 2005 \text { y tablas de vida de } \\
\text { la población }\end{array}$ \\
\hline IM a angina inestable & 0.0078 & HOPE trial 2000 (19) \\
\hline IM a IM & 0.0721 & ScHARR-TAG 2005 \\
\hline IM a diabetes & 0.0067 & HOPE trial 2000 \\
\hline IM a Acc.Cv & 0.0095 & ScHARR-TAG 2005 \\
\hline IM a falla cardiaca & 0.0230 & HOPE trial 2000 \\
\hline IM a muerte & 0.0217 & $\begin{array}{l}\text { ScHARR-TAG } 2005 \text { y tablas de vida de } \\
\text { la población }\end{array}$ \\
\hline Diabetes a angina inestable & 0.0021 & Doble riesgo de población sana \\
\hline Diabetes a IM & 0.0048 & Doble riesgo de población sana \\
\hline Diabetes a Acc.Cv & 0.0153 & Doble riesgo de población sana \\
\hline Diabetes a falla cardiaca & 0.0196 & Doble riesgo de población sana \\
\hline Diabetes a muerte & 0.0283 & Doble riesgo de población sana \\
\hline Acc. $\mathrm{Cv}$ a angina inestable & 0.0016 & Se asume que es igual que Acc.Cv a IM \\
\hline Acc.Cv a IM & 0.0016 & ScHARR-TAG 2005 \\
\hline Acc. $\mathrm{Cv}$ a diabetes & 0.0067 & Se asume que es igual que de IM a diabetes \\
\hline Acc.Cv a Acc.Cv & 0.2875 & ScHARR-TAG 2005 \\
\hline
\end{tabular}

Tabla 3. Riesgos relativos de los desenlaces de interés, medicamentos versus no intervención.

\begin{tabular}{|l|c|c|c|c|}
\hline Desenlace & $\begin{array}{c}\text { Diuréticos } \\
(\mathbf{D})\end{array}$ & $\begin{array}{c}\text { CCB } \\
(\mathbf{C})\end{array}$ & $\begin{array}{c}\text { BB } \\
(\mathbf{B})\end{array}$ & $\begin{array}{c}\text { ACEi/ARB } \\
(\mathbf{A})\end{array}$ \\
\hline Angina inestable & 0.893 & 0.881 & 0.984 & 1.01 \\
Infarto miocardio & 0.780 & 0.796 & 0.855 & 0.85 \\
Diabetes & 0.985 & 0.808 & 1.137 & 0.77 \\
Accidente cerebrovascular & 0.690 & 0.656 & 0.851 & 0.69 \\
Falla cardiaca & 0.530 & 0.731 & 0.761 & 0.65 \\
Muerte & 0.910 & 0.883 & 0.939 & 0.90 \\
\hline $\begin{array}{l}\text { CCB: Bloqueadores de los canales de calcio; BB: Betabloqueadores; ACEi/ARB: } \\
\text { inhibidores de la enzima convertidora de angiotensina/bloqueadores del receptor de } \\
\text { angiotensina. }\end{array}$
\end{tabular}

20 años hasta cumplir la expectativa de vida con el fin de evaluar las consecuencias en salud y costos de la enfermedad y del tratamiento. Para el caso base $98 \%$ de la cohorte de 65 años de edad, muere a la edad máxima.
Medición de la relación entre costos y efectos en salud

Se compararon los costos y resultados de cada una de las alternativas de tratamiento evaluadas mediante el cálculo de la razón de costo-efectividad. Con base en esto, se calculó la relación incremental de costo-efectividad entre las alternativas que resultaron dominantes.

La costo-efectividad fue calculada para cada medicamento a partir de un proceso simulación de 1000 repeticiones, donde se variaron los valores en el modelo. Para estimar los valores de los parámetros de probabilidad se decidió generar los valores de los parámetros a través de una distribución Beta (22). Para las variables de costos, los valores de los parámetros se generaron a través de la distribución Gamma. Para las variables de utilidad, también se decidió generar los valores a través de una distribución Beta.

Para definir la posibilidad que la intervención sea costoefectiva se tomó como umbral de costo-efectividad un valor estándar basado en el producto interno bruto (PIB) - per-cápita. Se definió una intervención como costo-efectiva para Colombia, si la razón de costo/efectividad está por debajo de tres veces el PIB, el cual se calculó en \$36 143 349, a partir de lo reportado para Colombia en 2011 por el Banco Mundial (21).

\section{Variabilidad e incertidumbre en la información}

Para evaluar el impacto de la incertidumbre en los supuestos usados en el análisis del caso de referencia respecto a la edad de inicio de la HTA, los riesgos basales de eventos cardiovasculares y el riesgo de presentar falla cardiaca y diabetes con el tratamiento, se realizaron análisis de sensibilidad univariados de tipo determinístico. Para evaluar el impacto de la incertidumbre en la estimación de costos se consideró un análisis de sensibilidad determinístico en el cual se varió la tarifa de contratación de servicios desde una tarifa mínima definida como ISS $2001+25 \%$, y una tarifa máxima definida como ISS $2001+48 \%$.

\section{Caso base}

\section{Resultados}

Los costos y los desenlaces medidos en QALYs de las estrategias bajo comparación se muestran en la Tabla 4, para el caso base definido anteriormente este análisis muestra que los diuréticos son menos costosos y más efectivos que la no intervención y que los betabloqueadores. Los calcioantagonistas tienen un incremento mínimo en QALYs con respecto a los diuréticos, pero su costo incremental es muy alto. Llama la atención que tanto el grupo de ARA/IECAS como el de diurético tienen una efectividad (QALYs) indistinguible, pero el costo asociado con ARA/IECAS es mucho mayor. La Figura 2 presenta el plano de costo-efectividad de los grupos de medicamentos contra la estrategia de no intervención.

\section{Análisis de subgrupos y de sensibilidad univariado no probabilístico}

En el resumen de los principales resultados de los diferentes análisis que se presentan en la Tabla 5 (a y b) se puede 
Tabla 4. Resultados del análisis de caso de referencia para hombres y mujeres.

\begin{tabular}{|c|c|c|c|}
\hline & $\begin{array}{l}\text { COSTO }(\mathrm{COP}) \\
\text { por persona }\end{array}$ & $\begin{array}{c}\text { EFECTO } \\
\text { (QALY* por persona) }\end{array}$ & $\begin{array}{c}\text { ICER * } \\
(\text { costo por QALY) }\end{array}$ \\
\hline \multicolumn{4}{|l|}{ Hombres } \\
\hline $\mathrm{D}$ & 6498624 & 9.24 & Opción de menor costo \\
\hline A & 13178919 & 9.24 & 6680295 , QALY igual \\
\hline $\mathrm{C}$ & 27774098 & 9.26 & 1063773700 \\
\hline B & 8563374 & 9.03 & Dominado \\
\hline NI & 6769607 & 8.80 & Dominado \\
\hline \multicolumn{4}{|l|}{ Mujeres } \\
\hline $\mathrm{D}$ & 6503235 & 9.49 & Opción de menor costo \\
\hline A & 13324399 & 9.49 & 6821 164, QALY igual \\
\hline $\mathrm{C}$ & 28340040 & 9.51 & 1091840250 \\
\hline B & 8626771 & 9.27 & Dominado \\
\hline NI & 6776312 & 9.03 & Dominado \\
\hline \multicolumn{4}{|c|}{$\begin{array}{l}\mathrm{D}=\text { diuréticos; } \mathrm{A}=\mathrm{ACEi} / \mathrm{ARB} ; \mathrm{C} \text { : bloqueadores de los canales de calcio; } \mathrm{B}=\text { betablo- } \\
\text { queadores; NI: no intervención. }\left({ }^{*}\right) \text { ICER: Relación incremental de costo-efectividad; } \\
\text { QALY: año de vida ajustado por calidad. }\end{array}$} \\
\hline
\end{tabular}

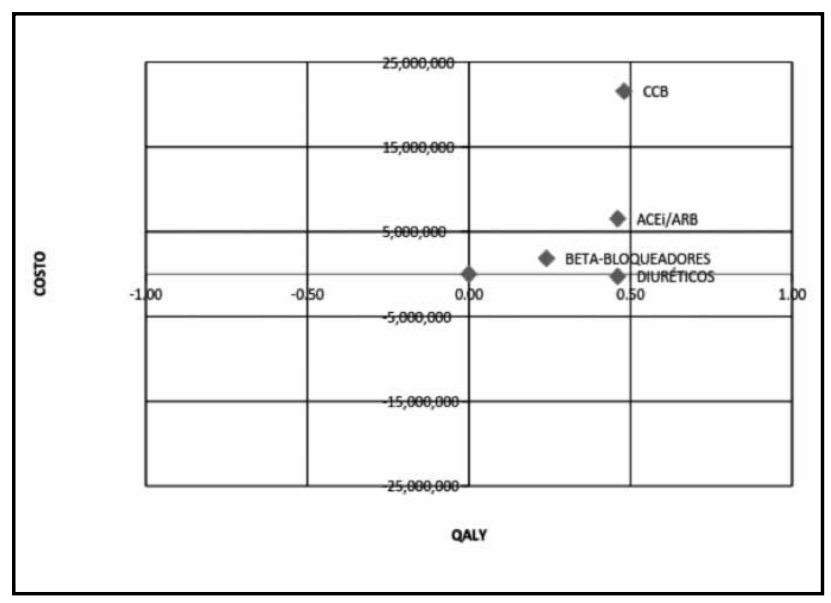

Figura 2. Plano de Costo-Efectividad para mujer de 65 años, $2 \%$ de riesgo anual de enfermedad cardiovascular, $1 \%$ de falla cardiaca, $1.1 \%$ de diabetes.

(Costo promedio ponderado del grupo de medicamentos, tasa de descuento $3 \%$, seguimiento por 20 años, y asumiendo que $20 \%$ de los sujetos serán intolerantes a ACEi y necesitarán un $A R B$ ).

QALY: año de vida ajustado por calidad; CCB: Bloqueadores de los canales de calcio; ACEi/ARB: inhibidores de la enzima convertidora de angiotensina/bloqueadores del receptor de angiotensina.

Tabla 5a. Resumen de resultados en los análisis de subgrupos y de sensibilidad.

\begin{tabular}{|c|c|}
\hline Escenario-Parámetros evaluados & Opción más costo/efectiva \\
\hline Caso base: hombre de 65 años, $2 \%$ de riesgo anual de enfermedad cardiovascular, $1 \%$ de falla cardiaca, $1.1 \%$ de diabetes & $\mathrm{D}$ \\
\hline Caso base: mujer de 65 años, $2 \%$ de riesgo anual de enfermedad cardiovascular, $1 \%$ de falla cardiaca, $1.1 \%$ de diabetes & $\mathrm{D}$ \\
\hline Grupo de edad de 45 años, otros supuestos sin cambios & $\mathrm{D}$ \\
\hline Grupo de edad de 55 años, otros supuestos sin cambios & $\mathrm{D}$ \\
\hline Grupo de edad de 75 años, otros supuestos sin cambios & $\mathrm{D}$ \\
\hline Riesgo anual menor de falla cardiaca $(0.5 \%)$ y mayor riesgo basal de diabetes $(3 \%)$, otros supuestos sin cambios & $\mathrm{D}$ \\
\hline Riesgo anual mayor de falla cardiaca ( $3 \%$ ) y mayor riesgo basal de diabetes $(3 \%)$, otros supuestos sin cambios & $\mathrm{D}$ \\
\hline
\end{tabular}

Tabla 5b. Resumen de resultados relevantes en los análisis de subgrupos y de sensibilidad.

\begin{tabular}{|c|c|}
\hline Escenario-Parámetros evaluados & Opción más costo/efectiva \\
\hline Bajo riesgo basal de falla cardiaca $(0.5 \%)$, otros supuestos sin cambios & $\mathrm{D}$ \\
\hline Alto riesgo basal de falla cardiaca (3\%), otros supuestos sin cambios & $\mathrm{D}$ \\
\hline Pérdida de utilidad por eventos adversos con diuréticos de $2 \%$, otros supuestos sin cambios & A \\
\hline Tasa de descuento $5 \%$, otros supuestos sin cambios & $\mathrm{D}$ \\
\hline Costo máximo de medicamentos, otros supuestos sin cambios & $\mathrm{D}$ \\
\hline Costo mínimo de medicamentos, otros supuestos sin cambios & $\mathrm{D}$ \\
\hline Costo mínimo de medicamentos, bajo riesgo de falla cardiaca $(0,5 \%)$ y alto riesgo relativo de diabetes $(3 \%)$ & A \\
\hline Costo mínimo de medicamentos, $5 \%$ de riesgo anual de enfermedad CV, bajo riesgo de falla cardiaca $(0,5 \%)$ y alto riesgo de diabetes $(3 \%)$ & A \\
\hline Sólo ARB en estimación del costo de grupo de ACEi/ARB, otros supuestos sin cambios & $\mathrm{D}$ \\
\hline Medicamento "indicador" por grupo, costo mínimo & $\mathrm{C}, \mathrm{A}$ \\
\hline Cambio a tarifas ISS $+25 \%$, otros supuestos sin cambios & $\mathrm{D}$ \\
\hline Cambio a tarifas ISS $+48 \%$, otros supuestos sin cambios & $\mathrm{D}$ \\
\hline
\end{tabular}


observar que la opción de diurético como mono-terapia es la más costo-efectiva, especialmente el tratamiento con tiazídicos (hidroclorotiazida). La principal diferencia entre las opciones de tratamiento está dada especialmente por el costo de adquisición de los medicamentos, dado que la efectividad medida en QALYs es bastante parecida entre las opciones de tratamiento con diuréticos y ACEi/ARB, y sólo discretamente mejor con calcioantagonistas.

Debe aclararse que el análisis es sensible al seleccionar un medicamento "indicador" por grupo, y utilizar el menor precio disponible en el mercado para ese medicamento; en esta situación, la opción de calcioantagonistas pasa a ser la más costo-efectiva, y los ACEi/ARB la segunda mejor opción. Como se aprecia también en la Tabla 5 (a y b), utilizando el precio promedio ponderado por frecuencia de uso en el grupo, los resultados de la evaluación económica son robustos tanto para hombres como para mujeres, y para grupos de edad diferentes al del caso base. De igual forma, aun en escenarios donde el riesgo basal de falla cardiaca es bajo, los diuréticos tiazídicos siguen siendo costo-efectivos. En situaciones de mayor riesgo de eventos cardiovasculares y de diabetes, donde los anticálcicos y los IECAs/ARA han probado ser muy efectivos, la opción de diurético sigue siendo más costo-efectiva. Una opción diferente (IECAs/ARAs) sólo pasa a ser la opción más costo-efectiva en estas situaciones especiales cuando se utiliza el menor precio del grupo de medicamentos.

La posibilidad de eventos adversos con diuréticos que afecten la calidad de la vida sólo haría que la decisión cambie cuando se asocian con una disminución en utilidades de $2 \%$ o más, y que las otras opciones no se asocien con eventos adversos que afecten la calidad de la vida, lo que parece ser un escenario poco probable. Finalmente, no hay diferencias en la opción más costo-efectiva cuando se utilizan cambios razonables en tarifas de contratación de servicios.

\section{Análisis de sensibilidad probabilístico}

Dados los resultados del análisis de referencia (caso base) que mostraron el diurético como la opción de menor costo, se decide mostrar en este análisis la distribución del costoefectividad incremental contra la opción de no intervención. Como se muestra en la Figura 3, el tratamiento antihipertensivo, en este caso con diurético, será dominante con respecto a no intervención en la mayoría de los escenarios, y en aquellos en los que no es dominante, es claramente costo-efectivo.

\section{Discusión}

El presente análisis muestra la evaluación económica en salud aplicada al tratamiento de la hipertensión leve a moderada recién diagnosticada, a través de la razón de costo efectividad incremental (ICER) para las principales intervenciones farmacológicas antihipertensivas de primera línea usadas como monoterapia (diuréticos, calcioantagonistas, betabloqueadores, inhibidores de enzima convertidora/ antagonistas del receptor de angiotensina), y contra una

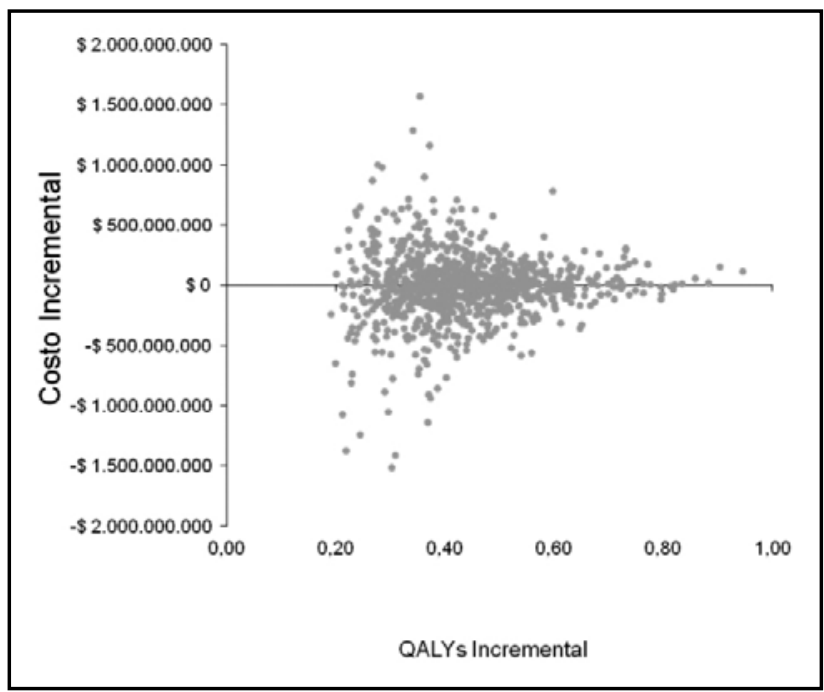

Figura 3. Análisis de costo-efectividad incremental de la terapia con diurético (versus no intervención). QALY: año de vida ajustado por calidad.

opción de no intervención.

En el escenario de caso base, la opción de tratamiento con diuréticos como monoterapia para la hipertensión leve a moderada recién diagnosticada, es la más costo-efectiva, especialmente con el uso de los tiazídicos (hidroclorotiazida), que en Colombia son los responsables de la prescripción de $98 \%$ de los diuréticos de venta en el país (23). Este análisis económico confirma hallazgos previos con respecto al perfil de costo-efectividad de estos medicamentos para el manejo de la HTA $(11,24)$, que puede llevar a ahorros sustanciales en el manejo de la HTA leve a moderada (25). La evaluación económica es robusta tanto para hombres como para mujeres, y para los grupos de edad de inicio de la HTA diferentes al del caso base. Aun en escenarios donde el riesgo basal de falla cardiaca es bajo, los diuréticos tiazídicos siguen siendo costo-efectivos, así como en situaciones de mayor riesgo de eventos cardiovasculares y de diabetes, donde los calcioantagonistas y los IECAs/ARA han probado ser muy efectivos (26-27).

En esta evaluación económica, las diferencias en costoefectividad entre los grupos de medicamentos está dada principalmente por el costo de adquisición de los medicamentos, dado que el efecto en la prevención de eventos cardiovasculares importantes es bastante parecida entre las opciones de tratamiento con diuréticos y ARA/IECA, y sólo discretamente mejor con calcioantagonistas. El análisis es sensible al precio de adquisición de los medicamentos; al seleccionar un medicamento "indicador" por grupo, y utilizar el menor precio disponible en el mercado para ese medicamento, la opción de calcioantagonistas puede llegar a ser la más costo-efectiva, y los IECAs/ARAs la segunda mejor opción. Esto lleva a pensar que disminuciones en el costo de adquisición de los medicamentos antihipertensivos, por referenciación internacional de precios u otros mecanismos, puede hacer cambiar estos resultados en un futuro. 
Aunque los resultados del análisis en los diferentes escenarios son consistentes (lo que muestra robustez en los resultados), el análisis económico realizado presenta limitaciones, la mayoría de ellas debidas a la escasez de información apropiada en varios aspectos: Primero, no hay mayor información disponible de efectividad en el grupo de edad entre 45 y 55 años, o en afrodescendientes, lo que necesitaría extrapolación cuidadosa de los resultados en estos grupos. Segundo, la efectividad de cada alternativa fue calculada con base en estudios donde muchos pacientes tuvieron necesidad de adicionar un segundo medicamento; aunque este dato es variable en la literatura puede llegar a ser hasta de 60\% (18). Se espera, sin embargo, que esto no llegue a cambiar los resultados finales en forma significativa si se asume que: 1 ) la probabilidad de requerir un tratamiento de segunda línea es igual para todos los grupos, y 2) si para todos los grupos de medicamentos se utilizarán medicamentos de segunda línea igual de efectivos. Si estos supuestos se aceptan, el esquema terapéutico de iniciar tratamiento con diurético y luego añadir un segundo medicamento, si se requiere, seguiría siendo la opción más costo-efectiva. Tercero, por falta de información, no fue posible incluir en este análisis el impacto de los retiros o abandonos de tratamiento, y la no adherencia al tratamiento con medicamentos. En caso que sea diferencial entre grupos de medicamentos, la efectividad calculada puede diferir de la obtenida en la vida real, y por tanto variar nuestras conclusiones. Sin embargo, el que los estudios incluidos para las estimaciones agrupadas de efectos del tratamiento, presenten sus resultados desde la filosofía de intención de tratar, asegura que sus resultados ya reflejan el efecto de estas posibles limitantes.

Una quinta limitación de la evaluación económica está asociada con la falta de estimaciones más precisas de los eventos adversos asociados a cada grupo de medicamentos, así como de su cuantificación e impacto sobre la calidad de vida. Si los costos asociados y la frecuencia de los eventos adversos asociados con los diuréticos son más altos, o afectan más la calidad de la vida que los eventos adversos que se asocian a los otros grupos de medicamentos, los resultados de este análisis pueden estar sesgados y beneficiar falsamente el tratamiento con diuréticos. Sin embargo, este inconveniente del análisis no es fácil de resolver ya que la metodología para incorporar en los análisis económicos la información relacionada con los eventos adversos (probabilidades, costos, y desenlaces) de los eventos adversos a medicamentos, no está bien desarrollada (28).

Sexto, el análisis de costo-utilidad se basa en utilidades tomadas de la literatura, reportadas en otros países con características culturales y de servicios muy diferentes a las colombianas. En la medida que los colombianos asignen un valor diferente a algunos de los estados de salud que hacen parte del modelo, los resultados del estudio podrían estar sesgado de manera no previsible. Sin embargo, las utilidades usadas en el cálculo de los QALY de este análisis se basan en estudios bien conducidos en el Reino Unido donde como es ampliamente conocido, se han hecho los estudios de medición de preferencias más válidos entre los evaluados para este análisis.

Finalmente, las limitaciones propias de los modelos de Markov, como es la de no guardar memoria de los estados previos al que el paciente está, es inherente en nuestro modelo. En la medida que esto sea importante en la transición entre estados de salud en las complicaciones de la HTA, esto pudiera afectar tanto los desenlaces estudiados, como los costos.

Este análisis económico tiene implicaciones para la práctica clínica colombiana. Los resultados de esta evaluación dieron soporte a la recomendación de prescribir diuréticos tiazídicos como terapia farmacológica de inicio en los pacientes promedio objeto de aplicación la guía colombiana (Recomendación 17). Aunque los datos de patrones de uso de medicamentos en Colombia son escasos, las observaciones preliminares (incluyendo la evaluación interna de usuarios potenciales de esta guía) en este escenario de práctica indican una muy baja tasa de uso de estos medicamentos. Este tipo de patrón se ha descrito en otros escenarios, incluidos países en desarrollo, constituyendo un sonado ejemplo de la distancia entre la práctica clínica y las recomendaciones basadas en evidencia. Ya otros autores han llamado la atención sobre los ahorros potenciales al sistema de salud, de adoptarse esta recomendación con una mayor frecuencia, como se menciona anteriormente. Por todas estas razones, la tasa de prescripción de diuréticos, producto de la recomendación soportada en esta evaluación puede ser uno de los indicadores más importantes para el seguimiento de la implementación y la adherencia a esta guía de práctica clínica.

En conclusión, esta evaluación económica mostró que en el escenario de caso base, la opción de tratamiento con diuréticos como monoterapia para la hipertensión leve a moderada recién diagnosticada, es la más costo-efectiva. La evaluación económica es robusta tanto para hombres como para mujeres, y para los grupos de edad de inicio de la HTA diferentes al del caso base. Aun en escenarios donde el riesgo basal de falla cardiaca es bajo, los diuréticos tiazídicos siguen siendo costo-efectivos, así como en situaciones de mayor riesgo de eventos cardiovasculares y de diabetes, donde los calcioantagonistas y los IECAs/ARA han probado ser muy efectivos. En esta evaluación económica, la diferencia entre los grupos de medicamentos está dada especialmente por el costo de adquisición de los medicamentos, dado que la efectividad en cuanto a la prevención de eventos cardiovasculares importantes es bastante parecida entre las opciones de tratamiento con diuréticos y ARA/IECA, y sólo discretamente mejor con calcioantagonistas.

\section{Financiación}

La realización de este estudio fue posible gracias a la financiación de COLCIENCIAS y del Ministerio de Salud de Colombia con la Pontificia Universidad Javeriana, y se desarrolló dentro del marco de la convocatoria 500 del 2009: Guía de Atención Integral para la "Prevención, diagnóstico, atención y seguimiento de pacientes con hipertensión arterial", con el fin de ser utilizado como un insumo en las recomen- 
daciones finales sobre tratamiento. Los autores no manifiestan ningún conflicto de interés con respecto al tema del manuscrito.

\section{Agradecimientos}

A Francis Ruiz en el National Institute for Clinical Excellence (NICE) del Reino Unido, por facilitar la versión del modelo económico que acompañó la Guía de HTA número 34 de NICE publicada en el 2006 para el Reino Unido (NICE no asume responsabilidad por la adaptación y aplicación del modelo en ningún país o región). A Joanne Lord, Universidad de Brunel, Reino Unido, por el desarrollo inicial del modelo económico. Al grupo desarrollador de Guía para la "Prevención, diagnóstico, atención y seguimiento de pacientes con hipertensión arterial". El desarrollo de la evaluación económica también fue posible gracias a los aportes de las siguientes personas e instituciones: Edgar Hernández MD, MSc, Daniel Isaza MD, Mónica López MD, y Mauricio Pineda MD, en la Fundación Cardio Infantil-Instituto de Cardiología, Bogotá; Dr. José Federico Saaibi, MD, en la Fundación Cardiovascular de Colombia, Bucaramanga; Drs. Manuel Urina, MD, MSc, y Ángel García, en el Hospital Universitario de San Ignacio, Bogotá.

\section{Referencias}

1. World Health Organization. Global health risks: mortality and burden of disease attributed to selected major risks. WHO Press, 2009; Disponible en: http://www. who.int/healthinfo/global_burden_disease/GlobalHealthRisks_report_full.pdf Citado: Enero 20 de 2015.

2. Lawes CM, Vander Hoom S, Rodgers A. Global burden of blood pressure related disease, 2001. Lancet 2008; 371: 1513-1518.

3. Global status report on non-communicable diseases, World Health Organization, 2010; WHO Press, Disponible en: http://www.who.int/nmh/publications/ ncd_report_full_en.pdf Citado: Enero 20 de 2015.

4. Hernandez R, Silva $\mathbf{H}$, Velasco M, et al. Hypertension in seven Latin American cities: the Cardiovascular Risk Factor Multiple Evaluation in Latin America (CARMELA) study.

5. Dennis R. Evaluación económica de la denervación simpática renal: simplicidad, todavía no. Rev Colomb Cardiol 2014; 152-153.

6. Mittal B, Singh A. Hypertension in the developing world: challenges and opportunities. Am J Kidney Dis 2010; 55: 590-598.

7. Pereira M, Lunet N, Azevedo A, Barros H. Differences in prevalence, treatment and control of hypertension between developing and developed countries. J Hypertens 2009; 27: 963-975.

8. Ministerio de Salud y Protección Social - Colciencias. Guía de práctica clínica. Hipertensión arterial temprana (HTA)- 2013 Guía No. 18. Disponible en: http://gpc. minsalud.gov.co/Documents/Guias-PDF/Recursos/HTA/ GPC_Completa_HTA. pdf Citado: Enero 20 de 2015

9. Ministerio de la protección social, Colciencias, Centro de estudios e investigación en salud de la Fundación Santa Fe de Bogotá, Escuela de salud pública de la Universidad de Harvard. Guía metodológica para el desarrollo de guías de atención integral en el sistema general de seguridad social en salud colombiano. Bogotá, Colombia 2010.

10. Redon J, Brunner HR, Ferri C, et al. Practical solutions to the challenge of uncontrolled hypertension. J Hypertens 2008; 26 (Sup 4): S1-S14.

11. National Clinical Guideline Centre (UK). Hypertension: The Clinical Management of Primary Hypertension in Adults: Update of Clinical Guidelines 18 and 34 [Internet].London: Royal College of Physicians (UK); 2011 Aug. Disponiblehttp:// www.ncbi.nlm.nih.gov/books/NBK83274/ Citado en: Enero 202015 www.nice. org.uk/guidance/CG127

12.Lipscomb J, Weinstein MC. \& Torrance GW. Time preference in Gold, J. E. Siegel, L. B. Russel \& M. C. Weinstein (Eds.), Cost-Effectiveness in Health and Medicine. New York: Oxford University Press 1996. P 214-246.
13. Departamento Administrativo Nacional de Estadística, DANE-Colombia. Defunciones por grupo de edad y sexo, según departamentos de ocurrencia y grupos de causas de defunción (lista Colombia 105 para la tabulación de mortalidad). Fecha de elaboración: 2008.

14. Tufts-New England Medical Center. Cost-Effectiveness Analysis Registry. (Internet).. Disponible en: https://research.tufts-nemc.org/cear4/ . Citado: Enero 20 de 2015

15. Instituto de Seguros S. Acuerdo No. 256 de 2001; Colombia Diciembre 19 de 2000. Disponible en: http://www.hrd.gov.co/documentos/facturacion/MANUAL\%20DE\%20FACTURACION\%202013/TARIFAS\%20ISS\%20-01\%20AC256. pdf . Citado: Enero 20 de 2015

16. Ministerio de la protección social. Decreto 4474 de 2010. Colombia; 29 de Noviembre de 2010. Disponible en: http://wsp.presidencia.gov.co/Normativa/Decretos/2010/Documents/Noviembre/29/dec447429112010.pdf. Citado en: Enero 20 de 2015.

17. Ward S, Lloyd Jones M, Pandor A, Holmes M, Ara R, Ryan A, Yeo W, Payne N. A systematic review and economic evaluation of statins for the prevention of coronary events. Health Technol Assess. 2007 Apr; 11 (14): 1-160.

18. Dahlof B, Sever PS, Poulter NR et al. Prevention of cardiovascular events with an antihypertensive regimen of amlodipine adding perindopril as required versus atenolol adding bendroflumethiazide as required, in the Anglo-Scandinavian Cardiac Outcomes Trial-Blood Pressure Lowering Arm (ASCOT-BPLA): a multicentre randomised controlled trial. Lancet 2005; 366: 895-906.

19. Perry HM, Davis BR, Price TR et al. Effect of treating isolated systolic hypertension on the risk of developing various types and subtypes of stroke: the Systolic Hypertension in the Elderly Program (SHEP). JAMA 2000; 284: 465-71.

20. Effects of ramipril on cardiovascular and microvascular outcomes in people with diabetes mellitus: results of the HOPE study and MICRO-HOPE substudy. Heart Outcomes Prevention Evaluation Study Investigators. Lancet. 2000 Jan 22; 355 (9200): 253-9. Erratum in: Lancet 2000 Sep 2; 356 (9232): 860.

21. The World Bank. GDP per cápita (Current US\$ ). 2013. Disponible en: http:// datos.bancomundial.org/indicador/NY.GDP.PCAP.CD. Citado en: February 20, 2013.

22. Briggs A, Claxton K \& Sculpher M, 2007. Decision Modelling for Health Economic Evaluations. Oxford: Oxford University Press.

23. Ministerio de la Protección Social. Listado de precios promedio y unidades en la cadena de comercialización de medicamentos. SISMED. Colombia 2010. Disponible en: http://www.sispro.gov.co/WebPublico/Consultas/ConsultarCNPMCadenaComercializacionCircu2yPA_028_2_1.aspx Citado en: Septiembre 28, 2012.

24. Stafilas PC, Sarafidis PA, Lasaridis An, et al. An economic evaluation of the 2003 ESH-ESC guidelines for the management of mild to moderate hypertension in Greece. AJH 2005; 18: 1233-1240.

25. Fretheim A, Aaserud M, Oxman A. The potential savings of using thiazides as first choice antihypertensive drug: cost-minimisation analysis. BioMed Central Health Services Research 2003; 3: 18.

26. Brenner BM, Cooper ME, de Zeeuw D, Keane WF, Mitch WE, Parving HH, Remuzzi G, Snapinn SM, Zhang Z, Shahinfar S. RENAL Study Investigators. Effects of losartan on renal and cardiovascular outcomes in patients with type 2 diabetes and nephropathy. N Engl J Med. 2001 Sep 20; 345 (12): 861-9.

27. Dahlöf B, Sever PS, Poulter NR, et al. ASCOT Investigators. Prevention of cardiovascular events with an antihypertensive regimen of amlodipine adding perindopril as required versus atenolol adding bendroflumethiazide as required, in the Anglo-Scandinavian Cardiac Outcomes Trial-Blood Pressure Lowering Arm (ASCOT-BPLA): a multicenter randomised controlled trial. Lancet. 2005 Sep 10-16; 366 (9489): 895-906.

28. Craig D, McDaid C, Fonseca T, Stock C, Duffy S, Woolacott N. Are adverse effects incorporated in economic models? An initial review of current practice. Health Technol Assess. 2009 Dec; 13 (62): 1-71, 97-181, iii. Review. 